\title{
Application of magnetic field hyperthermia and superparamagnetic iron oxide nanoparticles to HIV-I-specific T-cell cytotoxicity
}

This article was published in the following Dove Press journal:

International Journal of Nanomedicine

22 July 2013

Number of times this article has been viewed

\author{
James P Williams' \\ Paul Southern ${ }^{2}$ \\ Anya Lissina ${ }^{3}$ \\ Helen C Christian ${ }^{4}$ \\ Andrew K Sewell ${ }^{3}$ \\ Rodney Phillips ${ }^{1,5,6}$ \\ Quentin Pankhurst ${ }^{2}$ \\ John Frater ${ }^{1,5,6}$
}

'Nuffield Department of Clinical Medicine, John Radcliffe Hospital, Oxford, UK; ${ }^{2}$ Davy-Faraday Research Laboratory, Royal Institution of Great Britain, London, UK; ${ }^{3}$ Institute of Infection and Immunity, Cardiff University School of Medicine, Cardiff, UK; ${ }^{4}$ Department of Physiology, Anatomy and Genetics, University of Oxford, Oxford, UK; ${ }^{5}$ James Martin 21 st Century School, John Radcliffe Hospital, Oxford, UK; ${ }^{6}$ Oxford NIHR Biomedical Research Centre, Oxford, UK
Correspondence: John Frater Nuffield Department of Clinical Medicine, Peter Medawar Building for Pathogen Research, John Radcliffe Hospital, Oxford OXI 3SY, UK

Tel +44186527 I288

Email john.frater@ndm.ox.ac.uk

\begin{abstract}
The latent HIV-1 reservoir remains the major barrier to HIV-1 eradication. Although successful at limiting HIV replication, highly active antiretroviral therapy is unable to cure HIV infection, thus novel therapeutic strategies are needed to eliminate the virus. Magnetic field hyperthermia (MFH) generates thermoablative cytotoxic temperatures in target-cell populations, and has delivered promising outcomes in animal models, as well as in several cancer clinical trials. MFH has been proposed as a strategy to improve the killing of HIV-infected cells and for targeting the HIV latent reservoirs. We wished to determine whether MFH could be used to enhance cytotoxic T-lymphocyte (CTL) targeting of HIV-infected cells in a proof-of-concept study. Here, for the first time, we apply MFH to an infectious disease (HIV-1) using the superparamagnetic iron oxide nanoparticle FeraSpin R. We attempt to improve the cytotoxic potential of T-cell receptor-transfected HIV-specific CTLs using thermotherapy, and assess superparamagnetic iron oxide nanoparticle toxicity, uptake, and effect on cell function using more sensitive methods than previously described. FeraSpin R exhibited only limited toxicity, demonstrated efficient uptake and cell-surface attachment, and only modestly impacted T-cell function. In contrast to the cancer models, insufficient MFH was generated to enhance CTL killing of HIV-infected cells. MFH remains an exciting new technology in the field of cancer therapeutics, which, as technology improves, may have significant potential to enhance CTL function and act as an adjunctive therapy in the eradication of latently infected HIV-positive cells.
\end{abstract}

Keywords: nanoparticles, HIV-1, thermotherapy, cytotoxic T-cell

\section{Introduction}

Although highly active antiretroviral therapy (HAART) has revolutionized the management of HIV infection, it is not a cure. The major barrier to HAART-mediated $\mathrm{HIV}$ eradication is the formation of a latent reservoir of virally infected cells, ${ }^{1-4}$ which is the source of the rapid viral rebound seen in patients on stopping therapy. ${ }^{5-11}$ Patients must therefore have lifelong HAART, which is expensive and currently undeliverable to all who need it, especially in less industrialized regions, such as sub-Saharan Africa. ${ }^{12}$ Novel strategies need to be developed if HIV is to be eradicated.

Superparamagnetic iron oxide nanoparticles (SPIONs) are being trialed for use in the treatment of cancer by magnetic field hyperthermia (MFH), since enough heat can be produced by Néel relaxation on exposure to alternating magnetic fields to kill tumor cells by thermal ablation. ${ }^{13}$ On direct injection of SPIONs, hyperthermic temperatures were achieved in mouse models of breast cancer, leading to improved tumor control. ${ }^{14,15}$ SPIONs were well tolerated in human glioblastoma multiforme patients $^{16-18}$ and thermotherapeutic temperatures were achieved, leading to local tumor 
control and a doubled survival rate in patients. ${ }^{17,19} \mathrm{MFH}$ also induced a 4.5 -fold increase in survival time in a rat model of malignant glioma. ${ }^{20}$ In prostate cancer studies, a $44 \%-51 \%$ inhibition in growth of the aggressive MatLyLu tumor variant was observed on MFH treatment, with intratumoral temperatures of up to $70^{\circ} \mathrm{C}$ recorded..$^{21}$ SPIONs can also be targeted directly to tumors without direct intratumoral injection using SPION-conjugated monoclonal antibodies specific for cancer antigen. Significant therapeutic responses in mice with hamster blood transfusion 3477 human breast cancer xenografts were demonstrated, with tumors taking up to eight times longer to quintuple in size compared to controls. ${ }^{22}$

Cytotoxic T lymphocytes (CTLs) can bind to and kill HIV-infected cells and limit HIV replication. ${ }^{23-25}$ However, as disease progresses, HIV-specific CTLs lose their polyfunctional effector functions hierarchically, express exhaustion markers such as programmed death 1, T-cell immunoglobulin and musin domain containing protein 3 , and lymphocyte activation gene 3 , and have altered transcriptional profiles preventing optimal control of chronic infection. These functional defects are not restored by antiretroviral therapy (reviewed in Wherry ${ }^{26}$ ). ${ }^{27,28}$ Interestingly, hyperthermic temperatures enhance CTL cytotoxicity ${ }^{29,30}$ and stimulate CTL interferon-gamma release from CTLs. ${ }^{30}$

The application of SPIONs to HIV has been proposed as a technique for tracking HIV-specific CTLs to enhance vaccine delivery, as well as a possible therapeutic approach to targeting HIV-infected cells, ${ }^{31,32}$ since CTLs are permissive to SPION uptake. ${ }^{33-35}$ Resovist is a $60 \mathrm{~nm}$ carboxydextrancoated SPION with superior cellular uptake properties ${ }^{36-38}$ and a higher heating capability, or calculated intrinsic loss power, than other SPIONs, ${ }^{39}$ such as clinically available Endorem (sample VII). ${ }^{40}$ However, since Resovist is now commercially unavailable, Feraspin R, a closely related SPION formula with very similar properties to Resovist, ${ }^{41}$ was employed to study the feasibility of using MFH to enhance CTL killing of HIV-infected cells by two distinct mechanisms. The thermotherapeutic heat generated in cloned, Feraspin R-loaded autologous patient CTLs through alternating magnetic field exposure could either lead to the death of CTL-bound antigen-expressing target cells through direct thermal ablation or enhance their cytolytic CTL activity when these cells are adoptively transferred back into the patient. A recent study highlights the importance of CTLs in the targeting and clearance of latently HIV-infected cells, post viral reactivation with suberoylanilide hydroxamic acid (SAHA). ${ }^{42}$ Thus, in concert with antilatency compounds, thermotherapy could be used to target the cells that comprise the latent viral reservoir. Autologous functional, exhausted, or T-cell receptor (TCR)-transduced patient $\mathrm{CD} 8^{+} \mathrm{T}$ cells could be used for targeted thermotherapy, so long as they still bind to their targets through human leukocyte antigen (HLA) class I interactions. More sensitive methods to assay SPION uptake, $33,34,36-38,43$ toxicity, ${ }^{33,36-38,43}$ and effect on 868 TCR-transduced CTL function were developed, ${ }^{33-35}$ and SPION uptake was optimized using Lipofectamine and US Food and Drug Administration (FDA)-approved protamine sulfate. These optimized assays were then used to test if thermotherapy could impact HIV-1 CTL function.

\section{Materials and methods The 868 TCR system}

In this study, 868 TCR-transduced CTLs were used. We felt it important to utilize TCR-transduced cells rather than a CTL clone for several reasons. First, it is possible to grow large quantities of TCR-transduced cells, since these can come from many starting precursor cells, unlike a monoclonal T-cell population. Second, autologous TCR-transduced cells could be adoptively transferred into any patient with the relevant HLA allele. The $868 \mathrm{TCR}$ was deemed to be a good candidate for these studies, as it recognizes the immunodominant HLA A*0201-restricted, HIV p17 gag-derived epitope SLYNTVATL. Since the leucine residues at $\mathrm{p} 2$ and $\mathrm{p} 9$ in this epitope are essential for HIV gag trimerization and act as primary anchors for HLA-A2, they tend not to form escape mutants, since even conservative substitutions to valine result in extremely attenuated virus. Consequently, this epitope is believed to remain on the surface of all HLA $\mathrm{A} 2^{+}$HIV-infected cells in some form. ${ }^{44}$ Finally, a soluble recombinant form of the 868 TCR has the highest recorded natural affinity for its cognate antigen $\left(\mathrm{K}_{\mathrm{D}}<150 \mathrm{nM}\right)$, as measured by surface plasmon resonance, ${ }^{44}$ and will therefore maximize the length of interaction between target and effector cells for thermotherapy. We therefore reasoned that the 868 TCR-transduced $\mathrm{CD}^{+} \mathrm{T}$ cells would be an ideal starting point for our studies on MFH in HIV infection.

\section{Lentiviral transduction of $\mathrm{CD}^{+} \mathrm{T}$ cells}

Prior to transduction, primary $\mathrm{CD} 8^{+} \mathrm{T}$ cells were isolated from human peripheral blood mononuclear cells using anti-CD $8^{+}$ MACS microbeads (Miltenyi Biotec, Bergisch Gladbach, Germany), applied to a 24-well tissue culture plate at $10^{6}$ $\mathrm{CD}^{+}$cells/well in CK cell-culture media (R10 supplemented with $2.5 \%$ Cellkines [Helvetica Health Care, Geneva, Switzerland], $200 \mathrm{IU} / \mathrm{mL}$ interleukin [IL]-2 and $25 \mathrm{ng} / \mathrm{mL}$ IL-15 [PeproTech, Rocky Hill, NJ, USA]) and stimulated to 
divide by addition of anti-CD3/anti-CD28 antibody-coated microbeads (Life Technologies, Carlsbad, CA, USA) in a 6:1 beads:cells ratio for 24 hours at $37^{\circ} \mathrm{C}$ and $5 \% \mathrm{CO}_{2}$. Following the incubation period, half of the cell-culture media was removed and replaced by concentrated lentiviral supernatant encoding the 868 TCR. Efficiency of transduction of $\mathrm{CD}^{+}$ T cells (typically $>80 \%$ ) was assessed by flow cytometry using anti-CD8 antibody and cognate SL9/HLA A2 tetramer staining, following a 72-hour exposure to the lentivirus. If required, the transduced CTLs were further tetramer-sorted to obtain a clonal 868 TCR-expressing CTL line.

The lentivirus was generated by transfection of the packaging $293 \mathrm{~T}$ cell line with the 868 TCR-encoding lentiviral vector and the packaging plasmids $\mathrm{p} \Delta 8.91$ (coding for matrix and transcription proteins) and pMD2G (coding for envelope proteins) in the presence of Express-In transfection reagent (Thermo Fisher Scientific, Waltham, MA, USA). The original self-inactivating Lenti-SxW lentiviral vector (full name pHR'SINcPPT-SFFV-X-WPRE) used in this study was developed ${ }^{45}$ and kindly donated by Waseem Qasim (Molecular Immunology Unit, University College London). The 868 TCR construct, comprising the 868 TCR alpha and beta chains separated by an SGSG spacer sequence and 18 -amino acid $2 \mathrm{~A}$ fragment ${ }^{46}$ was initially assembled in a pUC19 shuttle vector, before being transferred to the Lenti-SxW lentiviral vector for transduction of primary $\mathrm{CD}^{+} \mathrm{T}$ cells.

\section{CTL expansion and characterization}

The 868 TCR-transduced primary $\mathrm{CD}^{+} \mathrm{T}$ cells were cultured as previously described for up to 3 weeks after initial stimulation. ${ }^{44}$ CTLs were stained with CD8 peridininchlorophyll-protein complex (PerCP; BD, Franklin Lakes, NJ, USA), live/dead fixable near-infrared dead-cell stain kit APC-Cy7 (Life Technologies) and SLYNTVATL9-tetramer phycoerythrin, all diluted in phosphate-buffered saline PBS (Life Technologies).

\section{CTL treatment with Feraspin $R$}

Feraspin R (stock concentration $10 \mathrm{mg} / \mathrm{mL}$ ) was custom made by Miltenyi Biotec. A total of $3 \times 10^{5} \mathrm{CTLs}$ per $100 \mu \mathrm{L}$ were cultured at $37^{\circ} \mathrm{C}, 5 \% \mathrm{CO}_{2}$ in IL-2 media (Roswell Park Memorial Institute 1640 media [SigmaAldrich, St Louis, MO, USA] supplemented with $25 \mathrm{ng} /$ mL IL-15 [Miltenyi Biotec], 100 IU/mL IL-2 [Roche, Basel, Switzerland], 10\% heat-inactivated fetal calf serum, $2 \mathrm{mM}$ glutamine, $0.1 \mathrm{mg} / \mathrm{mL}$ streptomycin, and $100 \mathrm{U} /$ $\mathrm{mL}$ penicillin [all Sigma-Aldrich]), with Feraspin R con- centrations of $0-1 \mathrm{mg} / \mathrm{mL}$ for 24 hours unless otherwise stated. Feraspin R was sonicated before resuspension in the cell-culture medium. For controls, untreated CTLs were cultured identically, except distilled water was added instead of Feraspin R. After incubation with or without Feraspin R, all cells were washed by centrifugation five times in PBS at $350 \mathrm{~g}$ to remove all unbound Feraspin R from the CTLs.

\section{Improving CTL uptake of Feraspin $\mathrm{R}$}

To improve nanoparticle uptake, CTLs were starved for 2 hours in PBS prior to incubation with nanoparticles. Other studies have demonstrated the transfection reagents protamine sulfate (Sigma-Aldrich) and Lipofectamine (Life Technologies) to increase cellular SPION uptake, so were prepared and added to the cell-culture medium at the concentrations previously described. ${ }^{33,47}$

\section{Microtomy and preparation for transmission electron microscopy}

A minimum of $10^{6}$ SPION-loaded or unloaded CTLs were fixed in $3 \% \mathrm{w} / \mathrm{v}$ paraformaldehyde, $0.05 \% \mathrm{v} / \mathrm{v}$ glutaraldehyde in PBS (pH 7.2) for 5 minutes at room temperature. The cells were embedded in LR Gold resin (London Resin Company, Reading UK) for electron microscopy by standard methods. Briefly, the cells were stained with uranyl acetate $(2 \% \mathrm{w} / \mathrm{v}$ in distilled water), dehydrated through increasing concentrations of ethanol (70\%-100\%) and embedded in LR Gold. Resin blocks were trimmed and then cut using a Reichert-Jung (Depew, NY, USA) Ultracut ultramicrotome with glass knife prepared using an LKB KnifeMaker (Pharmacia LKB Biotechnology, Piscataway, NJ, USA). Semithin $(0.5 \mu \mathrm{m})$ sections were mounted onto glass slides, stained with $1 \%$ toluidine (with 1\% borax), and examined to ensure the cells were in section. Ultrathin sections $(50-80 \mathrm{~nm})$ were mounted on nickel grids (Agar Scientific, Stansted, UK). Grids were then double-stained at room temperature with $2 \%$ uranyl acetate in methanol followed by lead citrate. The latter was done in a carbon dioxide-free environment, preventing the formation of a lead-based precipitate. Sections were rinsed briefly in distilled water and then dried thoroughly prior to examination using a JEOL (Tokyo, Japan) 1010 transmission electron microscope (TEM).

\section{Staining of Feraspin R-loaded CTLs}

For quantifying the percentage of $868 \mathrm{TCR}$-transduced CTLs that had taken up Feraspin R and had cell surface-bound 
Feraspin R, $10^{5}$ loaded and unloaded CTLs were stained with a live/dead near-infrared fixable dead-cell stain (APCCy7) and CD8-PerCP. Cells were resuspended in $200 \mu \mathrm{L}$ of Cytofix/Cytoperm fixation/permeabilization solution (BD) for 25 minutes, washed, resuspended in $200 \mu \mathrm{L}$ of Perm/ Wash buffer (BD), and washed again twice. Cells were stained with dextran-fluorescein isothiocyanate (FITC) (Stemcell Technologies, Vancouver, BC, Canada) in 10\% Perm/Wash solution, diluted in PBS for 25 minutes at room temperature. The cells were then washed twice more in Perm/ Wash and resuspended in 2\% formaldehyde (Sigma-Aldrich) and stored at $4^{\circ} \mathrm{C}$ until run on an LSR-II fluorescenceactivated cell-sorting (FACS) machine (BD).

\section{Quantification of Feraspin R uptake}

Magnetic susceptibility measurements were performed using a superconducting quantum interference device (SQUID; Quantum Design, San Diego, CA, USA). A change in sample position leads to a change in coil flux and thus circuit current, which can be used to infer the magnetic moment of the sample. Washed Feraspin R-loaded and unloaded CTLs (minimum of $3 \times 10^{5}$ cells per sample) were left to dry overnight at $37^{\circ} \mathrm{C}$ and $5 \% \mathrm{CO}_{2}$. Once dried, the samples were mounted in a gel capsule placed inside a plastic straw. SQUID voltages at different sample positions are averaged and taken at varying field strengths, up to 7 Tesla. By fitting the data to software models, the magnetic moment of Feraspin R-loaded CTLs can be calculated. The total content of iron per cell was calculated by comparing experimentally determined "emu" signals with the known emu/g value for Feraspin R and correcting for the amount of input cells.

\section{Nanoparticle-toxicity measurements}

A total of 50,000 loaded and unloaded CTLs were resuspended in $50 \mu \mathrm{L}$ of an antibody master mix, diluted in PBS with $1 \times$ volume of annexin $\mathrm{V}$ binding buffer. Cells were stained with the live/dead fixable near-infrared dead-cell stain kit, annexin V-FITC (Miltenyi Biotec), and CD8-PerCP for 25 minutes at $4^{\circ} \mathrm{C}$. The cells were washed twice in the PBS $1 \times$ annexin $\mathrm{V}$-binding buffer solution and were resuspended in PBS before FACS analysis. Annexin $\mathrm{V}^{+}$dead ${ }^{+}$gates were defined using Camptothecin-treated cells (50 nM; SigmaAldrich) as a positive control.

\section{Impact of temperature on CTL killing ability}

This assay is adapted from the fluorometric assessment of T-lymphocyte antigen-specific lysis (FATAL) assay, as first described by Sheehy et al. ${ }^{48} \mathrm{~T} 2$ (transporter associated with antigen processing-deficient) target cells were incubated (pulsed) with $10^{-7} \mathrm{M}$ of the HIV gag peptide SLYNTVATL for 1 hour at $37^{\circ} \mathrm{C}$, and then washed three times in PBS. The peptide-pulsed T2 cells were stained with carboxyfluorescein succinimidyl ester (Life Technologies) for 10 minutes and washed twice in PBS. A total of 50,000 peptide-pulsed cells were added per well in $50 \mu \mathrm{L}$ R10 media; 50,000 unpulsed T2 cells were stained with CellTracker Orange (Life Technologies) for 20 minutes, washed twice in R10, and added to each well. The 868 TCR-transduced CTLs were added at a 1:1 effector:target $(\mathrm{E}: \mathrm{T})$ ratio to a total volume of $150 \mu \mathrm{L}$. Two identical FATAL assays were performed, all samples in duplicate. One was incubated at $37^{\circ} \mathrm{C}$ and the other at $40^{\circ} \mathrm{C}$, both at $5 \% \mathrm{CO}_{2}$, and they were incubated for varying amounts of time. After incubation, the cells were washed and stained with CD8-PerCP and the live/dead fixable near-infrared dead-cell stain in PBS for 25 minutes at $4^{\circ} \mathrm{C}$. The cells were washed twice, resuspended in PBS and stored in the dark at $4^{\circ} \mathrm{C}$ until use. Carboxyfluorescein succinimidyl ester (FITC) versus CellTracker Orange (PE) FACS plots were gated on live CD8-negative cells, and decreases in the pulsed cell population (FITC) were measured as a percentage of the control-pulsed cell population, where no CTLs were added (percentage cytotoxicity as a percentage of control).

\section{Impact of Feraspin R loading on CTL killing ability}

FATAL assays were conducted as above, but using CTLs treated with increasing doses $(0-1 \mathrm{mg} / \mathrm{mL})$ of Feraspin $\mathrm{R}$ for 24 hours. CTLs were added at varying E:T ratios to the FATAL assay (0:1-1:10). Target T2 cells (American Type Culture Collection) were peptide pulsed with titrated $\left(10^{-12}\right.$ to $10^{-6} \mathrm{M}$ ) SLYNTVATL for 1 hour at $37^{\circ} \mathrm{C}$. The FATAL assays were incubated overnight at $37^{\circ} \mathrm{C}$ with $5 \% \mathrm{CO}_{2}$, and percentage of cytotoxicity as a percentage of control was measured, as above.

\section{Thermotherapy studies}

A magnetic alternating current hyperthermia system was used to expose SPIONs to a $1 \mathrm{MHz}$ alternating magnetic field with peak amplitude of $10.3 \mathrm{kA} / \mathrm{m}$, thus generating localized heat. To assess heat-related CTL death, CTLs incubated with $1 \mathrm{mg} / \mathrm{mL}$ Feraspin R for 24 hours treated with or without transfection agents or starvation were exposed to the alternating magnetic field for 1 hour. The solution temperature was kept constant at $37^{\circ} \mathrm{C}$ using an air warmer, to 
control for any bath-heating effect. Cell death was measured 1 hour and 24 hours postincubation to detect immediate and delayed cell death, using the toxicity protocol described above. The experiments were controlled using identically pretreated CTLs, which were incubated at $37^{\circ} \mathrm{C}$ for 1 hour, but not exposed to the alternating magnetic field. A further control assessed immediate and delayed cell death of Feraspin R-loaded CTLs by incubating the cells at known temperatures on a polymerase chain-reaction gradient (Bio-Rad Laboratories, Hercules, CA, USA) for 20 minutes. To assess the effect of Feraspin R thermotherapy on the enhancement of CTL killing, FATAL assays were incubated with and without alternating magnetic field exposure for 5 hours and the cells were stained, as mentioned above. All experiments were performed in duplicate.

\section{Results}

\section{Endocytic uptake of Feraspin R into 868-} TCR-transduced CTLs is dose-dependent and Feraspin $\mathrm{R}$ adheres to the cell surface

A dose-escalation experiment where 868 TCR-transduced CTLs were incubated with $0,0.1,0.5$, and $1 \mathrm{mg} / \mathrm{mL}$ Feraspin R was performed. The CTLs were viewed under a transmission electron microscope. After a 24-hour incubation, Feraspin $\mathrm{R}$ was shown to localize to the endosomal compartment (Figure 1) dose-dependently and to adhere to the cell surface (Figure 1B, lower arrow), a likely prerequisite for endosomal localization.
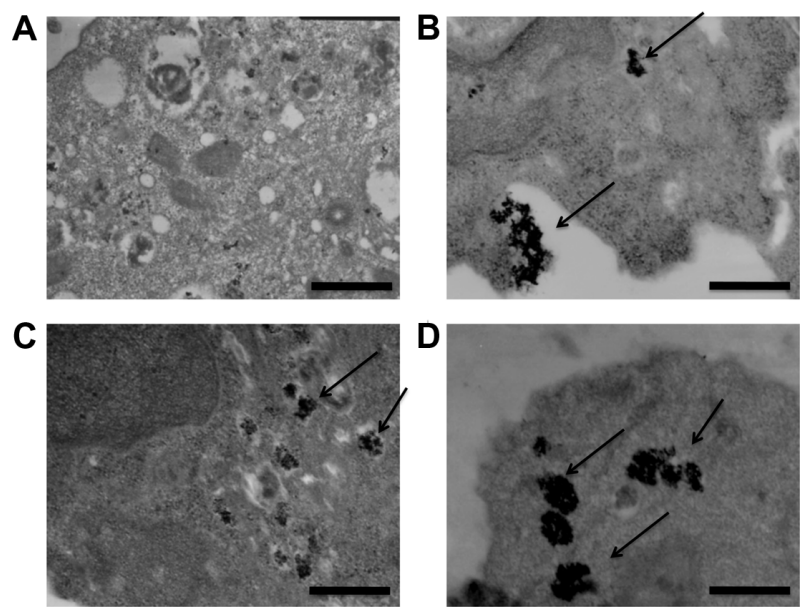

Figure I (A-D) Feraspin R is endocytosed dose-dependently by 868 TCRtransduced CTLs, and adheres to the cell surface. 868 TCR-transduced CTLs were incubated for up to 24 hours with (A) $0 \mathrm{mg} / \mathrm{mL}$, (B) $0.1 \mathrm{mg} / \mathrm{mL}$ (upper arrow shows internalized Feraspin R, lower arrow shows externally bound Feraspin R), (C) $0.5 \mathrm{mg} / \mathrm{mL}$, and (D) I mg/mL Feraspin R.

Notes: Uptake was observed by transmission electron microscopy (magnification 20,000x). Cell-surface attachment (B) was detected. Scale bars are $500 \mathrm{~nm}$. Abbreviations: TCR, T-cell receptor; CTL, cytotoxic T lymphocyte.
The amount of iron endocytosed into the cells was quantified by using a SQUID vibrating sample magnetometer. This uptake was time- ( $P=0.03$ by two-way analysis of variance [ANOVA] $)$ and concentration $(P=<0.001$ by twoway ANOVA)-dependent, with a maximum of $28.1 \mathrm{pg} / \mathrm{cell}$ measured after a 24-hour incubation of CTLs with $1 \mathrm{mg} / \mathrm{mL}$ Feraspin R (Figure 2A). This uptake was significantly enhanced using the transfection reagents Lipofectamine and the FDA-approved protamine sulfate $(P=0.04$ by two-way ANOVA), with a maximum of $53 \mathrm{pg} /$ cell achieved after incubating starved CTLs with a $1 \mathrm{mg} / \mathrm{mL}$ Feraspin R/protamine sulfate cocktail for 24 hours (Figure 2B). However, starvation did not have a significant effect on uptake over all conditions. This value is similar to that observed in other studies using Resovist analogues, albeit in different cell types and under different conditions. ${ }^{37,38}$ To determine the percentage of CTLs that endocytosed Feraspin R, an antidextran antibody conjugated to FITC, as previously reported by Beer et al, ${ }^{33}$ was used to stain the Feraspin R-treated CTLs intracellularly and extracellularly. The mean fluorescence-intensity change demonstrated that close to $100 \%$ of cells took up Feraspin R (Figure 2C). A generalized linear model statistical test demonstrated this effect to be dose-dependent whether using an intracellular or extracellular stain $(P<0.001)$. The interaction term between stain position (intracellular vs extracellular) and Feraspin R concentration was also significant $(P<0.001)$, indicating that at high concentrations of Feraspin R, significantly more nanoparticles were located intracellularly than extracellularly, but with the effect not significant at low concentrations.

\section{Feraspin $\mathrm{R}$ exhibits only limited toxicity to CTLs at concentrations tested}

Feraspin R toxicity was measured through a FACS staining protocol. Annexin V binds to phosphatidylserine, a phospholipid that is only found on the outer extracellular membrane leaflet on membrane flipping and a hallmark of apoptosis. Camptothecin-treated Feraspin R-loaded CTLs were used as positive controls to define and gate out dead and apoptotic cells from the live-cell population at each Feraspin $\mathrm{R}$ incubation concentration (Figure 3A). A general linear model statistical test demonstrated significant $(P=0.042)$ toxicity with increasing Feraspin R dose after 24 hours (Figure 3B), although the actual absolute toxicity values were small. After washing off the nanoparticles and further incubation of CTLs in R10 media, there was a significant interaction between the percentage of living cells and time after CTL washing $(P=0.003$, generalized linear model $)$, with a trend 

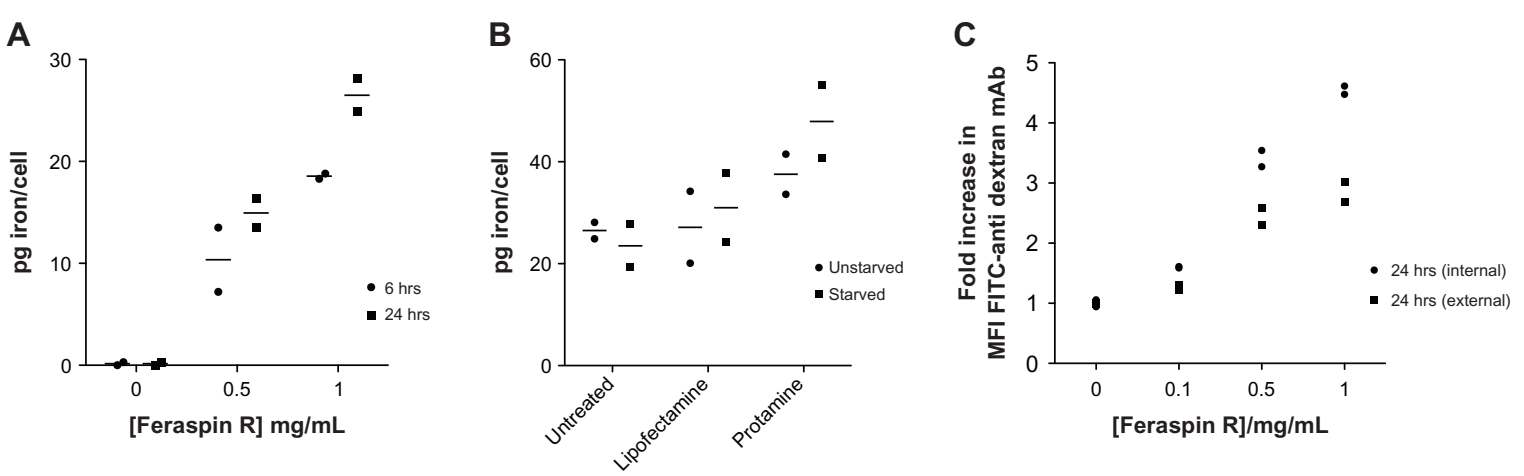

Figure 2 (A-C) Endocytotic uptake of Feraspin R into 868 TCR-transduced CTLs is efficient and enhanced with transfection reagents. (A) 868 TCR-transduced CTLs were incubated with varying concentrations of Feraspin R for 6 hours or 24 hours, and cell-associated Feraspin R was quantified by SQUID. (B) Cell-culture Feraspin R media was supplemented with the transfection reagents Lipofectamine or protamine sulfate for 24 hours and quantified by SQUID. (C) Feraspin R loaded CTLs were stained intracellularly or extracellularly with an antidextran antibody FITC to determine intracellular and extracellular Feraspin R associations.

Notes: The fold increase in MFI for intracellularly (circles figure $2 \mathrm{C}$ ) and extracellularly (squares) located Feraspin R was plotted against the incubation concentration of Feraspin R. All experiments were performed in duplicate.

Abbreviations: TCR, T-cell receptor; CTL, cytotoxic T lymphocyte; SQUID, superconducting quantum interference device; MFI, mean fluorescence intensity; FITC, fluorescein isothiocyanate; pg, picograms.

to increasing viability for CTLs over time for both treatment groups. Also, the minor toxic effects of $1 \mathrm{mg} / \mathrm{mL}$ Feraspin $\mathrm{R}$ were still observed after washing $(P=<0.001$, generalized linear model) compared to untreated sample, but the trend was for this effect to be lost over time (Figure 3C). Transfection-reagent treatment and starvation had no effect on cell viability (Figure 3D).

\section{Insufficient heat generated by Feraspin $\mathrm{R}$ to kill CTLs}

Firstly, CTLs were incubated with Feraspin R at $1 \mathrm{mg} / \mathrm{mL}$ for 24 hours with and without transfection reagents in an alternating magnetic field for 1 hour (Figure 4A and B). Cell-death and apoptosis markers were analyzed by FACS staining 1 hour and 24 hours after magnetic field exposure.
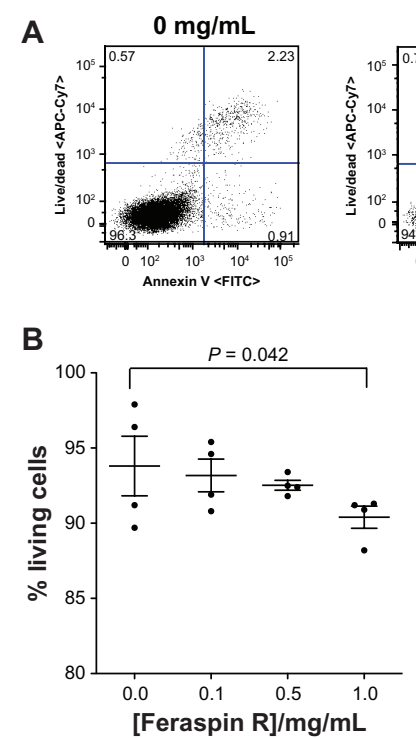

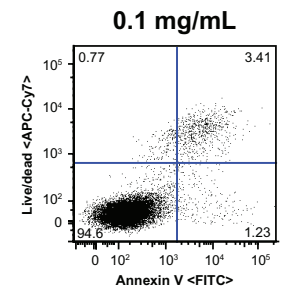

C

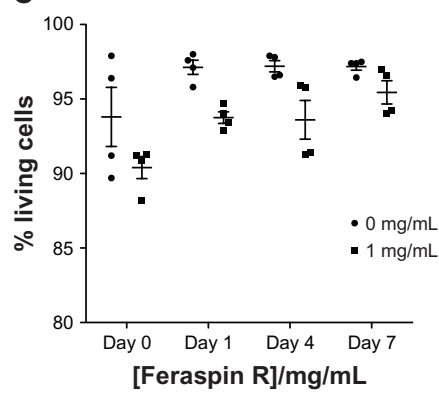

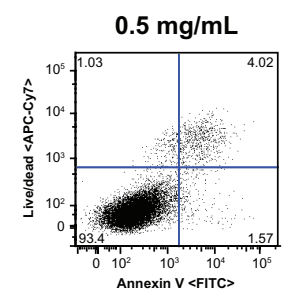
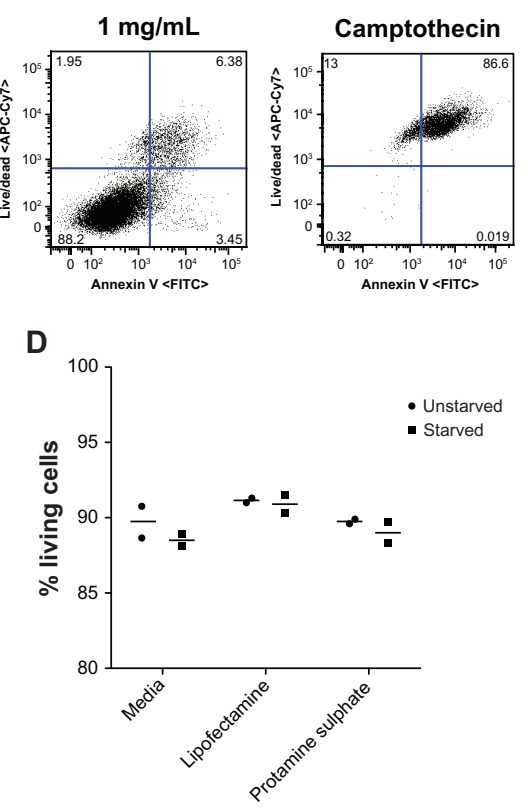

Figure 3 (A-D) Feraspin R exhibits only limited toxicity to 868 TCR-transduced CTLs at the concentrations tested. 868 TCR-transduced CTLs were incubated for 24 hours with increasing doses of Feraspin R. (A) Live CD8 ${ }^{+} \mathrm{T}$ cells were gated, and apoptotic (annexin $\mathrm{V}^{+}$) and dead (allophycocyanin cytochrome $7^{+}$) cell gates were determined using a camptothecin-treated control for all doses of Feraspin R-treated CTLs. (B) Dead and apoptotic cells were identified and the population of annexin V-negative live cells were calculated as a percentage of total gated $\mathrm{CD}^{+}$cells (\% living cells) in quadruplicate. (C) CTLs incubated for 24 hours with and without I mg/mL Feraspin $\mathrm{R}$ were washed and cultured for a further week. The percentage of living cells at days I, 4, and 7 were determined in quadruplicate. (D) CTLs were incubated with Feraspin R in the presence of transfection reagents, and the percentage of living cells post-24-hour incubation was determined in duplicate.

Abbreviations: TCR, T-cell receptor; CTL, cytotoxic T lymphocyte; FITC, fluorescein isothiocyanate; CD, cluster of differentiation. 
A

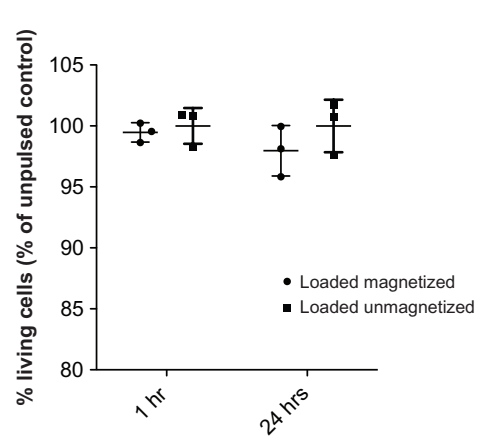

B

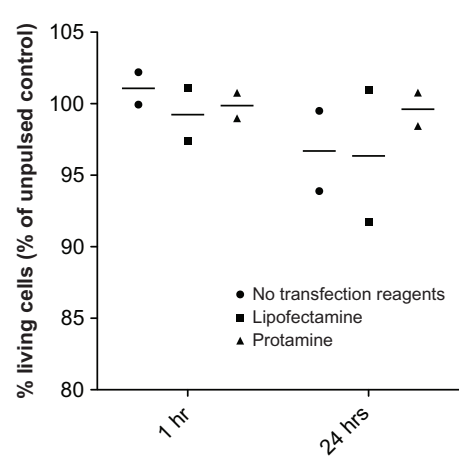

C

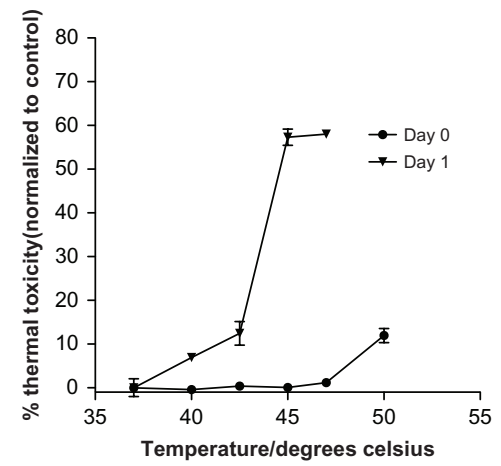

Figure $4(\mathbf{A}-\mathbf{C})$ Insufficient heat is generated in Feraspin R-loaded 868 TCR-transduced CTLs for thermal ablation. CTLs treated for 24 hours with I mg/mL in the absence (A) or presence (B) of transfection reagents were exposed to an alternating magnetic field for I hour. Unmagnetized loaded CTL controls were included. The percentage of living cells (as in Figure 3) was measured I hour and 24 hours postmagnetization to detect immediate and delayed cell death. (A and B) were performed in triplicate and duplicate, respectively. (C) Immediate and delayed cell death of Feraspin R-loaded CTLs at various known incubation temperatures, in triplicate.

Abbreviations: TCR, T-cell receptor; CTL, cytotoxic T lymphocyte.

The above experiments were all controlled using loaded CTLs that were incubated for the same time and at the same bath temperature, but were not exposed to an alternating magnetic field. Also, controls were included where Feraspin R-loaded CTLs were incubated at known temperatures. No significant increase and no trend in cell death after alternating magnetic field exposure could be determined by twoway ANOVA, even after transfection-reagent treatment (Figure 4B), despite a 20-minute temperature pulse of above $44.5^{\circ} \mathrm{C}$ being enough to kill $50 \%$ of Feraspin R-loaded CTLs 24 hours later (Figure $4 \mathrm{C}$ ).

A

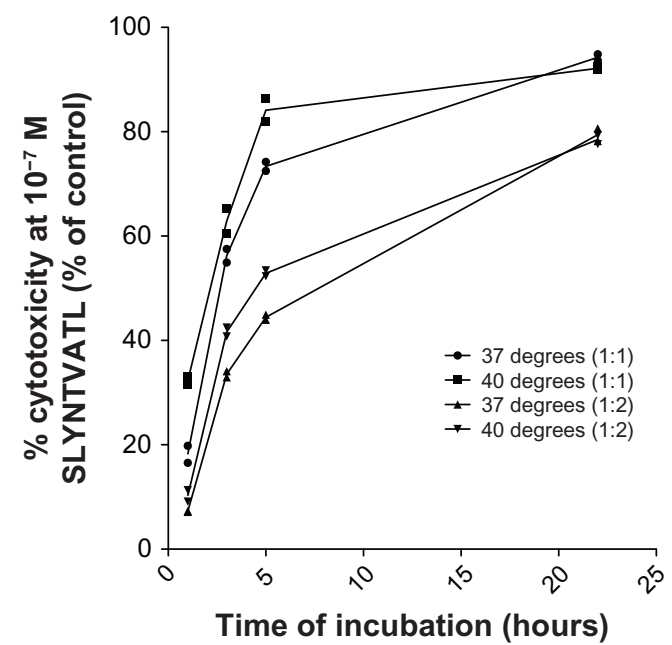

\section{CTLs exhibit increased cytotoxicity at $40^{\circ} \mathrm{C}$ compared to $37^{\circ} \mathrm{C}$}

The FATAL assay, as demonstrated by Sheehy et al in 2001, ${ }^{48}$ was used to investigate CTL cytotoxicity at different temperatures. FATAL killing assays were carried out at $37^{\circ} \mathrm{C}$ and $40^{\circ} \mathrm{C}$ using two E:T ratios of 1:1 and 1:2. After logtransforming time, the generalized linear model demonstrated a statistically significant increase in the percentage of cytotoxicity of $\mathrm{CTL}$ at $40^{\circ} \mathrm{C}$ compared to $37^{\circ} \mathrm{C}(P=0.017)$ across both E:T ratios, and cytotoxicity increased with higher E:T ratios $(P=<0.001)$ and $\log ($ time $)(P<0.001)$ (Figure 5A).

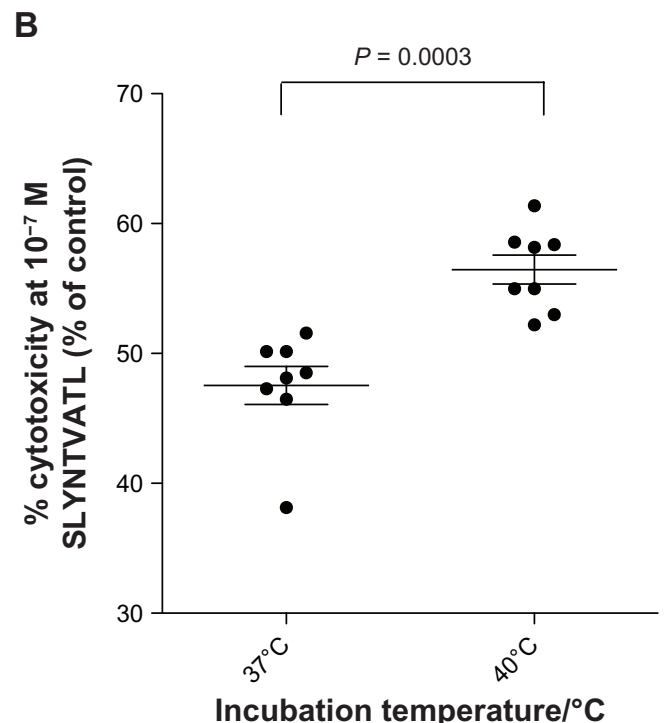

Figure 5 (A and B) Increased 868 TCR-transduced CTL cytotoxicity at $40^{\circ} \mathrm{C}$ compared with $37^{\circ} \mathrm{C}$. (A) Fluorometric assessment of T-lymphocyte antigen-specific lysis killing assays were performed in duplicate and analyzed over time using SLYNTVATL pulsed T2 cells and 868 TCR CTLs at I:I or I:2 effector:target ratios at different temperatures $\left(37^{\circ} \mathrm{C}\right.$ or $\left.40^{\circ} \mathrm{C}\right)$. Decreases in the peptide-pulsed carboxyfluorescein succinimidyl ester-positive cell population (fluorescein isothiocyanate) were measured, as a percentage of the control-pulsed cell population, where no CTLs were added (percentage cytotoxicity as a percentage of control). (B) Percentage cytotoxicity (as a percentage of control) using $10^{-7} \mathrm{M}$ SLYNTVATL-pulsed T2 cells was determined after a 5-hour incubation as above, with eight replicates at $37^{\circ} \mathrm{C}$ or $40^{\circ} \mathrm{C}$. Abbreviations: TCR, T-cell receptor; CTL, cytotoxic T lymphocyte. 
The biggest difference in percentage of cytotoxicity was observed to be after a 5-hour incubation (Figure 5A). The percentage cytotoxicity of CTLs increased from $48 \%$ to $57 \%$ (n $=8, P=0.0003$ ) after 5 hours of incubation (Figure $5 \mathrm{~B}$ ), and this increased cytotoxicity was independent of direct heat-induced target-cell death (data not shown).

\section{Feraspin R modestly affects CTL cytotoxicity}

The FATAL assay was used to investigate the effects of Feraspin R on CTL killing ability. The killing ability of CTLs decreased by 5\%-10\% on addition of the lowest concentration $(0.1 \mathrm{mg} / \mathrm{mL})$ of Feraspin $\mathrm{R}$ across all $\mathrm{E}: \mathrm{T}$ ratios tested, and then only modestly decreased on addition of more nanoparticles (Figure 6A). The generalized linear model demonstrated a significant decrease in CTL cytotoxicity on increasing nanoparticle concentration $\left(\log _{10}\right.$ [Feraspin $\mathrm{R}$ concentration +0.001$])$, across all $\mathrm{E}: \mathrm{T}$ ratios $(P=0.007)$. CTLs loaded for 24 hours with $1 \mathrm{mg} / \mathrm{mL}$ Feraspin $\mathrm{R}$ were then tested for their ability to kill peptide-pulsed T2 cells at different SLYNTVATL peptide concentrations, and half-maximal effective concentration $\left(\mathrm{EC}_{50}\right)$ values were calculated. There was less than a log difference in killing ability between loaded and unloaded CTLs, with respective $\mathrm{EC}_{50}$ values of $1.34 \times 10^{-8} \mathrm{M}$ and $2.59 \times 10^{-9} \mathrm{M}$ (Figure $6 \mathrm{~B}$ ). The generalized linear model demonstrated that Feraspin $\mathrm{R}$ significantly decreased CTL killing ability across all peptide concentrations $(P=0.028)$ after the percentagecytotoxicity data was transformed using the equation logit $(5+\%$ cytotoxicity $) / 100$.

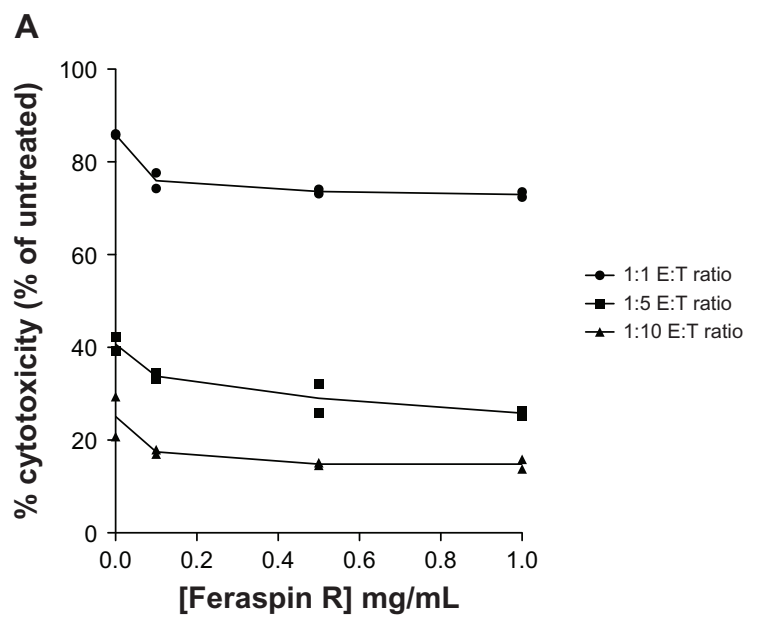

\section{Insufficient heat is generated by Feraspin $R$ to enhance CTL cytotoxicity}

FATAL assays using Feraspin R-loaded and unloaded CTLs were performed during 5-hour exposures to an alternating magnetic field. The experiments were performed in duplicate and were controlled with identical FATAL assays, not exposed to the magnetic field, but incubated at the same bath temperature of $37^{\circ} \mathrm{C}$. A decrease in killing ability was seen on nanoparticle addition; however, no significant increase in killing ability of loaded magnetized cells versus loaded unmagnetized cells was observed by two-way ANOVA (Figure 7). Therefore, not enough heat was generated by the cell-associated Feraspin R to impact CTL cytotoxicity, as seen above.

\section{Discussion}

SPIONs and magnetic field hyperthermia exhibit exciting new therapeutic and diagnostic applications in nanomedicine. To date, these have been focused on cancer, but here we present the first attempts to apply this technology to an infectious disease - HIV. There are similarities between tumor biology and that of the HIV latent reservoir, and a number of current HIV-eradication strategies are based on a cancer model. ${ }^{49}$ For example, in a landmark study by Archin et al, ${ }^{49}$ the chemotherapy drug vorinostat (SAHA) induces in vivo HIV transcription from patients' latently HIV-1 infected cells. This can cause infected cell death through viral cytopathic effects, or CTL clearance of these cells through HIV antigen presentation, theoretically reducing the HIV reservoir. ${ }^{42}$ Thus, there is hope that a remission

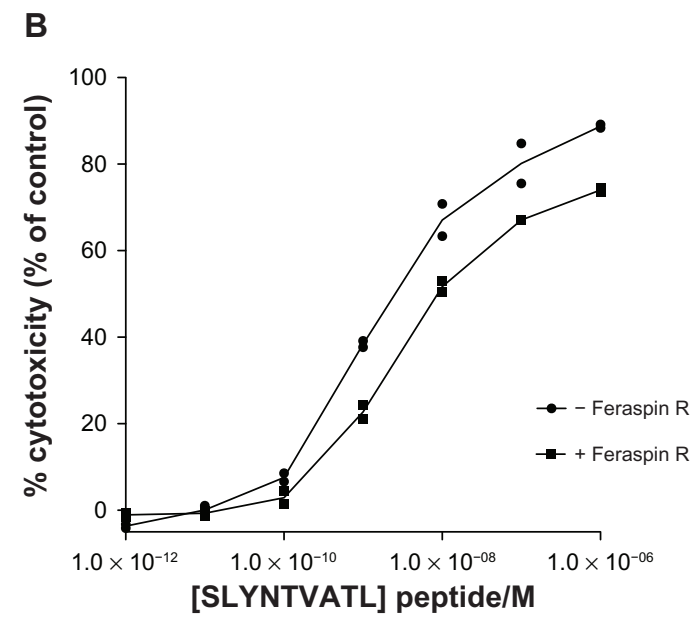

Figure 6 (A and B) 868 TCR-transduced CTLs kill less efficiently when treated with Feraspin R. FATAL assays were performed using (A) CTLs pretreated with various concentrations of Feraspin R for 24 hours at various effector:target (E:T) ratios, and (B) CTLs treated for 24 hours with I mg/mL Feraspin R at various concentrations of SLYNTVATL peptide-pulsed T2 cells.

Note: All FATAL assays were incubated overnight and performed in duplicate.

Abbreviations: TCR, T-cell receptor; CTL, cytotoxic T lymphocyte; FATAL, fluorometric assessment of T-lymphocyte antigen-specific lysis; E:T, effector:target. 


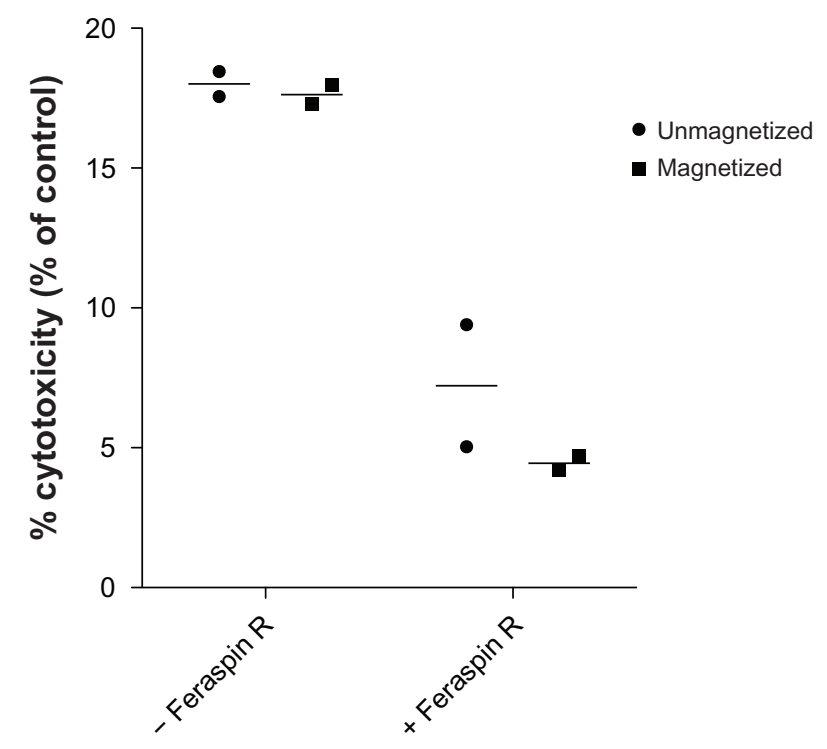

Figure 7 Insufficient heat is generated inside 868 TCR-transduced CTLs for enhancement of cytotoxicity.

Notes: Fluorometric assessment of T-lymphocyte antigen-specific lysis assays were performed using Feraspin R-loaded or unloaded CTLs at a I:I effector:target ratio and were incubated with $10^{-7} \mathrm{M}$ SLYNTVATL-treated T2 cells with or without alternating magnetic field exposure for 5 hours. The percentage cytotoxicity of CTLs (as a percentage of control) was calculated as before. All experiments were performed in duplicate.

Abbreviations: TCR, T-cell receptor; CTL, cytotoxic T lymphocyte.

phase from HAART may be achievable, similar to that of chemotherapy in cancer, whereby patients can either be cured, or control the residual HIV burden in the absence of therapy. Here, we wished to apply SPION technology to HIV-specific T-cells to enhance their function and test in a proof-of-concept study as to whether MFH was applicable to HIV infection. Although T-cells frequently bind to HIVinfected CD4 target cells and release interferon gamma, this is not consistently associated with killing. These responses have been coined passenger T-cell responses, in contrast to the driver responses, which result in target-cell death. ${ }^{50}$ We wished to enhance T-cell function through thermotherapy by two possible mechanisms. We hypothesized that raising the temperature of T-cells to the equivalent of physiological fever $\left(40^{\circ} \mathrm{C}\right)$ would increase function and cytotoxicity. In addition, we tested the hypothesis that thermotherapy might increase the temperature of the T-cell and its bound target cell to directly induce cell death.

Nanoparticle toxicity, uptake, and effect of cell function is not well characterized for CTLs. We describe improved methods to detect Feraspin R toxicity, uptake, and effect on cell function, as well as exploring the two potential therapies described above. Previous studies have shown endosomal SPION uptake into CTLs for the purpose of T-cell tracking through the body by magnetic resonance imaging (MRI) ${ }^{33,35}$ and T-cell magnetically mediated immunothermotherapy for melanoma. ${ }^{34}$ As seen in other studies, we observed endosomally located Feraspin R, and the uptake increases dose-dependently and on transfection-agent treatment (Figures 1 and 2). SQUID has advantages over other ironquantification methods, such as inductive coupled plasma atomic absorption spectroscopy and atomic absorption spectroscopy ${ }^{38}$ which likely overestimate iron levels by not distinguishing between nonparamagnetic and paramagnetic iron. Iron concentrations are calculated indirectly in the colorimetric potassium thiocyanate method, ${ }^{34}$ on the assumption that full oxidation or reduction of a dissolved sample occurs. A concentration of $53 \mathrm{pg} /$ cell (Figure 2B) was measured in our study poststarvation and on protamine sulfate treatment, a much higher value than that observed by Beer et al, ${ }^{33}$ but our results matched those observed using Resovist analogues in other cell types. ${ }^{36,37,43} \mathrm{~A}$ limitation of Lipofectamine is that it is not licensed for use in vivo; however, protamine sulfate is FDA-approved for use in vivo, and will be useful in future studies where high cellular SPION uptake is crucial.

The percentage uptake of Feraspin R into cells was measured using an antidextran monoclonal antibody, as used by Beer et al. ${ }^{33}$ Close to $100 \%$ of CTLs took up nanoparticles, negating the need for magnetic enrichment of nanoparticleloaded cells (Figure 2C). We demonstrated through TEM (Figure 1) and FACS (Figure 2) staining that Feraspin R binds CTLs extracellularly, an issue that may not have been taken into account in previous studies. We therefore advise caution in quantifying intracellular uptake of Feraspin $\mathrm{R}$ and define our SQUID results as representing cell-associated iron.

We used a sensitive FACS-based method to detect dead and apoptotic cells for assessing Feraspin R toxicity. More cells can be analyzed and counted, and the results are much less subjective than for a trypan blue assay that demonstrated no Resovist toxicity to CTLs in a previous study. ${ }^{33}$ Around $5 \%$ toxicity $(P=0.042)$ was observed at $1 \mathrm{mg} / \mathrm{mL}$ Feraspin $\mathrm{R}$ (Figure $3 \mathrm{~A}$ and $\mathrm{B}$ ) and may have been caused by reactive oxygen species generated in the Fenton reaction, which cause lipid peroxidation, and DNA damage ${ }^{51,52}$ when the carboxydextran coat of Resovist is degraded, exposing free iron in acidic lysosomal cellular compartments, ${ }^{53}$ or a recently described novel mechanism of nonapoptotic cell death - ferroptosis. ${ }^{54}$

The effect of nanoparticle uptake on CTL killing ability is poorly characterized. Interferon-gamma secretion enzymelinked immunosorbent assays previously used for this purpose $^{34}$ do not directly measure CTL killing ability, and chromium-release assays ${ }^{33,35}$ have a number of disadvantages, 
including a high spontaneous leakage rate, effect of ${ }^{51} \mathrm{Cr}$ on the effector cell population, the fact that certain cell types cannot be labeled properly, insensitivity, and safety issues associated with the use of gamma radiation. ${ }^{48}$ In the FATAL assay, there is $40 \times$ less spontaneous leakage, a more accurate assessment of the E:T ratio can be demonstrated, and lower levels of CTL-specific lysis. ${ }^{48}$ We demonstrated using this more sensitive method that CTL killing ability decreased on Feraspin R incubation (Figure 6). This may be explained by extracellularly bound nanoparticle complexes on the CTL cell surface blocking binding interactions between peptidepresenting T2 cells and the 868-TCR Feraspin R located in lytic granules interfering or poisoning cytolytic enzymes, or low-level Feraspin R toxicity of CTLs. $\mathrm{EC}_{50}$ values for Feraspin R-loaded $\left(1.34 \times 10^{8} \mathrm{M}\right)$ and unloaded $\left(2.59 \times 10^{9} \mathrm{M}\right)$ CTLs across a range of peptide titrations were established for the first time. Previously, no significant differences in CTL killing ability between loaded and unloaded CTLs had been described, but this could be due to much lower concentrations of Resovist, different SPIONs, and less sensitive or direct methods being employed for detection. ${ }^{33-35}$

Despite it not being necessary for the Feraspin R-loaded CTLs to retain native killing function in this study, some retention of CTL homing might be preferred. Previous studies have shown through MRI scanning that SPION-labeled cancerspecific CTLs traffic to tumors, and be differentially targeted on repeat injection. ${ }^{35}$ Autoimmune lymphocytes loaded with SPIONs were also shown to locate to the beta-islet cells of the pancreas in nonobese diabetic mice. ${ }^{55}$ This suggests that some CTL homing functions are retained on SPION treatment.

We were unable to detect increased cytotoxicity in magnetized Feraspin R-loaded CTLs compared to unmagnetized cells (Figures 4 and 7). However temperatures of above $44.5^{\circ} \mathrm{C}$ for 20 minutes were enough to kill $50 \%$ of Feraspin R-loaded CTLs and CTLs demonstrated higher cytotoxic potential at $40^{\circ} \mathrm{C}$ compared to $37^{\circ} \mathrm{C}$ (Figure 5). This suggests that despite transfection-reagent treatment and uptake optimization, insufficient Feraspin $\mathrm{R}$ was engulfed per cell to cause cell death, or adequate thermotherapeutic enhancement of CTL function using the magnetic alternating current hyperthermia system. Since a recent publication demonstrated that hyperthermia increased HIV replication, any future experiments assessing the thermotherapeutic effects of nanoparticles in targeting of HIV-infected cells must be performed in the presence of HAART. ${ }^{56}$ However, this study did not assess the potentially beneficial effect of thermotherapy to increase CTL killing of HIV antigenpresenting cells.
A recently published fluorescence-based technology for measuring intracellular temperature could be useful in evaluating how much more Feraspin $\mathrm{R}$ per cell is needed to achieve thermotherapeutic temperatures, how the heat penetrates through the cell membrane, and if it is evenly distributed across the cell. ${ }^{57}$ Recent data suggest that cellcycle stage and cell density are important for maximizing nanoparticle uptake. ${ }^{58}$ Although CTLs were treated with Feraspin $\mathrm{R}$ at the same cell concentrations and CTLs were split 1:124 hours before treatment, the above issues were not specifically addressed, and could account for any disparity in repeat SQUID-uptake results. However, it is unlikely that these factors would cause sufficient increased SPION uptake for there to be a thermotherapeutic effect and enhancement of CTL function. Future work involving the optimization of uptake conditions for clinically available SPIONs and the analysis of SPION uptake and MFH on heat-shock protein, T-cell receptor, or cytolytic granule-protein expression may prove useful to fully understand the role of thermotherapy on CTL function.

MRI has detected the migration of SPION-loaded melanoma-specific CTLs to tumors at a resolution of three cells per voxel in mice in vivo ${ }^{35}$ and trafficking of dendritic cells to melanomas in patients. ${ }^{35,59}$ Antilatency drugs such as SAHA (vorinostat) can reexpress latent HIV from HAART-treated patient cells. ${ }^{60,61}$ Assuming viral antigens are expressed after treatment, it may be possible to use SPIONloaded HIV-specific CTLs to identify the elusive and muchdebated location of the latent HIV viral reservoir.

In summary, we have been able to show that HIV-specific cytotoxic T-cells will bind to and take up SPIONs, and that this does not greatly impact cell physiology or cell death. We were also able to confirm that HIV-specific CTLs kill more efficiently at higher temperatures, equivalent to those induced by a fever. However, in this study we were unable to achieve SPION-associated cell death through thermotherapy. This remains an exciting and novel application of nanotechnology. As SPION uptake and incubation methods are improved, SPIONs with superior uptake profiles and higher heating capabilities created, and the strength of the applied alternating magnetic field increased, SPION CTL thermotherapy may have significant potential to enhance CTL function and act as an adjunctive therapy in both the imaging and eradication of latently HIV-infected cells.

\section{Acknowledgments}

We would like to thank James Ussher, Chris Willberg, and Nicole Yager for valuable advice in FACS analysis, Saman 
Ghannadzadeh in the Department of Physics, University of Oxford, for help with and use of the SQUID, and Helen Fryer for help with statistical analysis.

\section{Disclosure}

The authors report no conflicts of interest in this work.

\section{References}

1. Chun TW, Finzi D, Margolick J, Chadwick K, Schwartz D, Siliciano RF. In vivo fate of HIV-1-infected T cells: quantitative analysis of the transition to stable latency. Nat Med. Dec 1995;1(12):1284-1290.

2. Chun TW, Stuyver L, Mizell SB, et al. Presence of an inducible HIV-1 latent reservoir during highly active antiretroviral therapy. Proc Natl Acad Sci U SA. 1997;94(24):13193-13197.

3. Finzi D, Hermankova M, Pierson T, et al. Identification of a reservoir for HIV-1 in patients on highly active antiretroviral therapy. Science. 1997;278(5341):1295-1300.

4. Wong JK, Hezareh M, Günthard HF, et al. Recovery of replicationcompetent HIV despite prolonged suppression of plasma viremia. Science. 1997;278(5341):1291-1295.

5. Dybul M, Daucher M, Jensen MA, et al. Genetic characterization of rebounding human immunodeficiency virus type 1 in plasma during multiple interruptions of highly active antiretroviral therapy. $J$ Virol. 2003;77(5):3229-3237.

6. Harrigan PR, Whaley M, Montaner JS. Rate of HIV-1 RNA rebound upon stopping antiretroviral therapy. AIDS. 1999;13(8):F59-F62.

7. Davey RT Jr, Bhat N, Yoder C, et al. HIV-1 and T cell dynamics after interruption of highly active antiretroviral therapy (HAART) in patients with a history of sustained viral suppression. Proc Natl Acad Sci U SA. 1999;96(26):15109-15114.

8. Zhang L, Chung C, Hu BS, et al. Genetic characterization of rebounding HIV-1 after cessation of highly active antiretroviral therapy. $J$ Clin Invest. 2000;106(7):839-845.

9. Joos B, Fischer M, Kuster H, et al. HIV rebounds from latently infected cells, rather than from continuing low-level replication. Proc Natl Acad Sci U SA. 2008;105(43):16725-16730.

10. Kijak GH, Simon V, Balfe P, et al. Origin of human immunodeficiency virus type 1 quasispecies emerging after antiretroviral treatment interruption in patients with therapeutic failure. J Virol. 2002;76(14): 7000-7009.

11. Chun TW, Davey RT Jr, Ostrowski M, et al. Relationship between pre-existing viral reservoirs and the re-emergence of plasma viremia after discontinuation of highly active anti-retroviral therapy. Nat Med. 2000;6(7):757-761.

12. Hecht R, Bollinger L, Stover J, et al. Critical choices in financing the response to the global HIV/AIDS pandemic. Health Aff (Millwood). 2009;28(6):1591-1605.

13. Pankhurst QA, Connolly J, Jones SK, Dobson J. Applications of magnetic nanoparticles in biomedicine. J Phys D Appl Phys. 2003; 36(13):R167-R181.

14. Jordan A, Scholz R, Wust P, et al. Effects of magnetic fluid hyperthermia (MFH) on C3H mammary carcinoma in vivo. Int J Hyperthermia. 1997; 13(6):587-605.

15. Zhai Y, Xie H, Gu H. Effects of hyperthermia with dextran magnetic fluid on the growth of grafted $\mathrm{H} 22$ tumor in mice. Int J Hyperthermia. 2009;25(1):65-71.

16. Muller S. Magnetic fluid hyperthermia therapy for malignant brain tumors - an ethical discussion. Nanomedicine. 2009;5(4):387-393.

17. Maier-Hauff K, Rothe R, Scholz R, et al. Intracranial thermotherapy using magnetic nanoparticles combined with external beam radiotherapy: results of a feasibility study on patients with glioblastoma multiforme. J Neurooncol. 2007;81(1):53-60.

18. Jordan A, Maier-Hauff K. Magnetic nanoparticles for intracranial thermotherapy. J Nanosci Nanotechnol. 2007;7(12):4604-4606.
19. Maier-Hauff K, Ulrich F, Nestler D, et al. Efficacy and safety of intratumoral thermotherapy using magnetic iron-oxide nanoparticles combined with external beam radiotherapy on patients with recurrent glioblastoma multiforme. J Neurooncol. 2011;103(2):317-324.

20. Jordan A, Scholz R, Maier-Hauff K, et al. The effect of thermotherapy using magnetic nanoparticles on rat malignant glioma. J Neurooncol. 2006;78(1):7-14.

21. Johannsen M, Thiesen B, Jordan A, et al. Magnetic fluid hyperthermia (MFH) reduces prostate cancer growth in the orthotopic Dunning R3327 rat model. Prostate. 2005;64(3):283-292.

22. DeNardo SJ, DeNardo GL, Miers LA, et al. Development of tumor targeting bioprobes ( ${ }^{111}$ In-chimeric L6 monoclonal antibody nanoparticles) for alternating magnetic field cancer therapy. Clin Cancer Res. 2005;11(19 Pt 2):7087s-7092s.

23. Schmitz JE, Kuroda MJ, Santra S, et al. Control of viremia in simian immunodeficiency virus infection by CD8+ lymphocytes. Science. 1999; 283(5403):857-860.

24. Hersperger AR, Migueles SA, Betts MR, Connors M. Qualitative features of the HIV-specific CD8+T-cell response associated with immunologic control. Curr Opin HIV AIDS. May 2011;6(3):169-173.

25. Walker BD, Chakrabarti S, Moss B, et al. HIV-specific cytotoxic T lymphocytes in seropositive individuals. Nature. 1987;328(6128): 345-348.

26. Wherry EJ. T cell exhaustion. Nat Immunol. 2011;12(6):492-499.

27. Migueles SA, Weeks KA, Nou E, et al. Defective human immunodeficiency virus-specific CD8+ T-cell polyfunctionality, proliferation, and cytotoxicity are not restored by antiretroviral therapy. J Virol. 2009; 83(22):11876-11889.

28. Kalams SA, Goulder PJ, Shea AK, et al. Levels of human immunodeficiency virus type 1-specific cytotoxic T-lymphocyte effector and memory responses decline after suppression of viremia with highly active antiretroviral therapy. J Virol. 1999;73(8):6721-6728.

29. Smith JB, Knowlton RP, Agarwal SS. Human lymphocyte responses are enhanced by culture at 40 degrees C. J Immunol. 1978;121(2): 691-694.

30. Mace TA, Zhong LW, Kokolus KM, Repasky EA. Effector CD8+ $\mathrm{T}$ cell IFN-gamma production and cytotoxicity are enhanced by mild hyperthermia. Int J Hyperthermia. 2012;28(1):9-18.

31. Murthy SK. Nanoparticles in modern medicine: state of the art and future challenges. Int J Nanomedicine. 2007;2(2):129-141.

32. Dodd $\mathrm{CH}, \mathrm{Hsu} \mathrm{HC}$, Chu WJ, et al. Normal T-cell response and in vivo magnetic resonance imaging of T cells loaded with HIV transactivatorpeptide-derived superparamagnetic nanoparticles. J Immunol Methods. 2001;256(1-2):89-105.

33. Beer AJ, Holzapfel K, Neudorfer J, et al. Visualization of antigenspecific human cytotoxic $\mathrm{T}$ lymphocytes labeled with superparamagnetic iron-oxide particles. Eur Radiol. 2008;18(6):1087-1095.

34. Iida H, Takayanagi K, Nakanishi T, et al. Preparation of human immune effector T cells containing iron-oxide nanoparticles. Biotechnol Bioeng. 2008;101(6):1123-1128.

35. Kircher MF, Allport JR, Graves EE, et al. In vivo high resolution three-dimensional imaging of antigen-specific cytotoxic T-lymphocyte trafficking to tumors. Cancer Res. 2003;63(20):6838-6846.

36. Mailander V, Lorenz MR, Holzapfel V, et al. Carboxylated superparamagnetic iron oxide particles label cells intracellularly without transfection agents. Mol Imaging Biol. 2008;10(3):138-146.

37. Metz S, Bonaterra G, Rudelius M, Settles M, Rummeny EJ, Daldrup-Link HE. Capacity of human monocytes to phagocytose approved iron oxide MR contrast agents in vitro. Eur Radiol. 2004; 14(10):1851-1858.

38. Matuszewski L, Persigehl T, Wall A, et al. Cell tagging with clinically approved iron oxides: feasibility and effect of lipofection, particle size, and surface coating on labeling efficiency. Radiology. 2005;235(1):155-161.

39. Kallumadil M, Tada M, Nakagawa T, Abe M, Southern P, Pankhurst QA. Suitability of commercial colloids for magnetic hyperthermia. J Magn Magn Mater. 2009;321(10):1509-1513. 
40. Hergt R, Andra W, d'Ambly CG, et al. Physical limits of hyperthermia using magnetite fine particles. IEEE Trans Magn. 1998;34(5): 3745-3754.

41. Ludwig F, Wawrzik T, Yoshida T, et al. Optimization of magnetic nanoparticles for magnetic particle imaging. IEEE Trans Magn. 2012; 48(11):3780-3783.

42. Shan L, Deng K, Shroff NS, et al. Stimulation of HIV-1-specific cytolytic T lymphocytes facilitates elimination of latent viral reservoir after virus reactivation. Immunity. 2012;36(3):491-501.

43. Yeh CH, Hsiao JKH, Wang JL, Shen F. Immunological impact of magnetic nanoparticles (ferucarbotran) on murine peritoneal macrophages. J Nanopart Re. 2010;12(1):151-160.

44. Varela-Rohena A, Molloy PE, Dunn SM, et al. Control of HIV-1 immune escape by CD8 T cells expressing enhanced T-cell receptor. Nat Med. 2008;14(12):1390-1395.

45. Demaison C, Parsley K, Brouns G, et al. High-level transduction and gene expression in hematopoietic repopulating cells using a human immunodeficiency (correction of immunodeficiency) virus type 1-based lentiviral vector containing an internal spleen focus forming virus promoter. Hum Gene Ther. 2002;13(7):803-813.

46. Yang S, Cohen CJ, Peng PD, et al. Development of optimal bicistronic lentiviral vectors facilitates high-level TCR gene expression and robust tumor cell recognition. Gene Ther. 2008;15(21):1411-1423.

47. Arbab AS, Yocum GT, Rad AM, et al. Labeling of cells with ferumoxides-protamine sulfate complexes does not inhibit function or differentiation capacity of hematopoietic or mesenchymal stem cells. NMR Biomed. 2005;18(8):553-559.

48. Sheehy ME, McDermott AB, Furlan SN, Klenerman P, Nixon DF. A novel technique for the fluorometric assessment of $\mathrm{T}$ lymphocyte antigen specific lysis. J Immunol Methods. 2001;249(1-2):99-110.

49. Archin NM, Liberty AL, Kashuba AD, et al. Administration of vorinostat disrupts HIV-1 latency in patients on antiretroviral therapy. Nature. 2012;487(7408):482-485.

50. Zafiropoulos A, Barnes E, Piggott C, Klenerman P. Analysis of 'driver' and 'passenger' CD8+ T-cell responses against variable viruses. Proc Biol Sci. 2004;271 Suppl 3:S53-S56.
51. Yang CY, Hsiao JK, Tai MF, et al. Direct labeling of hMSC with SPIO: the long-term influence on toxicity, chondrogenic differentiation capacity, and intracellular distribution. Mol Imaging Biol. 2011;13(3):443-451.

52. Soenen SJ, Himmelreich U, Nuytten N, Pisanic TR 2nd, Ferrari A, De Cuyper M. Intracellular nanoparticle coating stability determines nanoparticle diagnostics efficacy and cell functionality. Small. 2010; 6(19):2136-2145.

53. Tycko B, Maxfield FR. Rapid acidification of endocytic vesicles containing alpha 2-macroglobulin. Cell. 1982;28(3):643-651.

54. Dixon SJ, Lemberg KM, Lamprecht MR, et al. Ferroptosis: an iron-dependent form of nonapoptotic cell death. Cell. 2012;149(5): 1060-1072.

55. Moore A, Sun PZ, Cory D, Hogemann D, Weissleder R, Lipes MA. MRI of insulitis in autoimmune diabetes. Magn Reson Med. 2002; 47(4):751-758.

56. Roesch F, Meziane O, Kula A, et al. Hyperthermia stimulates HIV-1 replication. PloS Pathog. 2012;8(7):e1002792.

57. Okabe K, Inada N, Gota C, Harada Y, Funatsu T, Uchiyama S. Intracellular temperature mapping with a fluorescent polymeric thermometer and fluorescence lifetime imaging microscopy. Nat Commun. 2012;3:705.

58. Kim JA, Aberg C, Salvati A, Dawson KA. Role of cell cycle on the cellular uptake and dilution of nanoparticles in a cell population. Nat Nanotechnol. 2011;7(1):62-68.

59. de Vries IJ, Lesterhuis WJ, Barentsz JO, et al. Magnetic resonance tracking of dendritic cells in melanoma patients for monitoring of cellular therapy. Nat Biotechnol. 2005;23(11):1407-1413.

60. Contreras X, Schweneker M, Chen CS, et al. Suberoylanilide hydroxamic acid reactivates HIV from latently infected cells. J Biol Chem. 2009;284(11):6782-6789.

61. Archin NM, Espeseth A, Parker D, Cheema M, Hazuda D, Margolis DM. Expression of latent HIV induced by the potent HDAC inhibitor suberoylanilide hydroxamic acid. AIDS Res Hum Retroviruses. 2009;25(2):207-212.
International Journal of Nanomedicine

\section{Publish your work in this journal}

The International Journal of Nanomedicine is an international, peerreviewed journal focusing on the application of nanotechnology in diagnostics, therapeutics, and drug delivery systems throughout the biomedical field. This journal is indexed on PubMed Central, MedLine, CAS, SciSearch $\AA$, Current Contents ${ }^{\circledR} /$ Clinical Medicine,

\section{Dovepress}

Journal Citation Reports/Science Edition, EMBase, Scopus and the Elsevier Bibliographic databases. The manuscript management system is completely online and includes a very quick and fair peer-review system, which is all easy to use. Visit http://www.dovepress.com/ testimonials.php to read real quotes from published authors. 